\title{
THE
}

6-8-2015

\section{Simulation of Observed PCBs and Pesticides in the Water Column during the North Atlantic Bloom Experiment}

Lin Zhang

Louis Thibodeaux

Lee Jones

Rainer Lohmann

University of Rhode Island, rlohmann@uri.edu

Follow this and additional works at: https://digitalcommons.uri.edu/gsofacpubs

The University of Rhode Island Faculty have made this article openly available.

Please let us know how Open Access to this research benefits you.

This is a pre-publication author manuscript of the final, published article.

Terms of Use

This article is made available under the terms and conditions applicable towards Open Access

Policy Articles, as set forth in our Terms of Use.

\section{Citation/Publisher Attribution}

Lin Zhang, Louis Thibodeaux, Lee Jones and Rainer Lohmann (2015). " Simulation of Observed PCBs and Pesticides in the Water Column during the North Atlantic Bloom Experiment. "Environmental Science \& Technology, 49,(23), 13760-13767.

Available at: http://dx.doi.org/10.1021/acs.est.5b00223

This Article is brought to you for free and open access by the Graduate School of Oceanography at DigitalCommons@URI. It has been accepted for inclusion in Graduate School of Oceanography Faculty Publications by an authorized administrator of DigitalCommons@URI. For more information, please contact digitalcommons-group@uri.edu. 


\section{Supporting Information for}

Simulation of observed PCBs and pesticides in the water column during the North Atlantic Bloom Experiment.

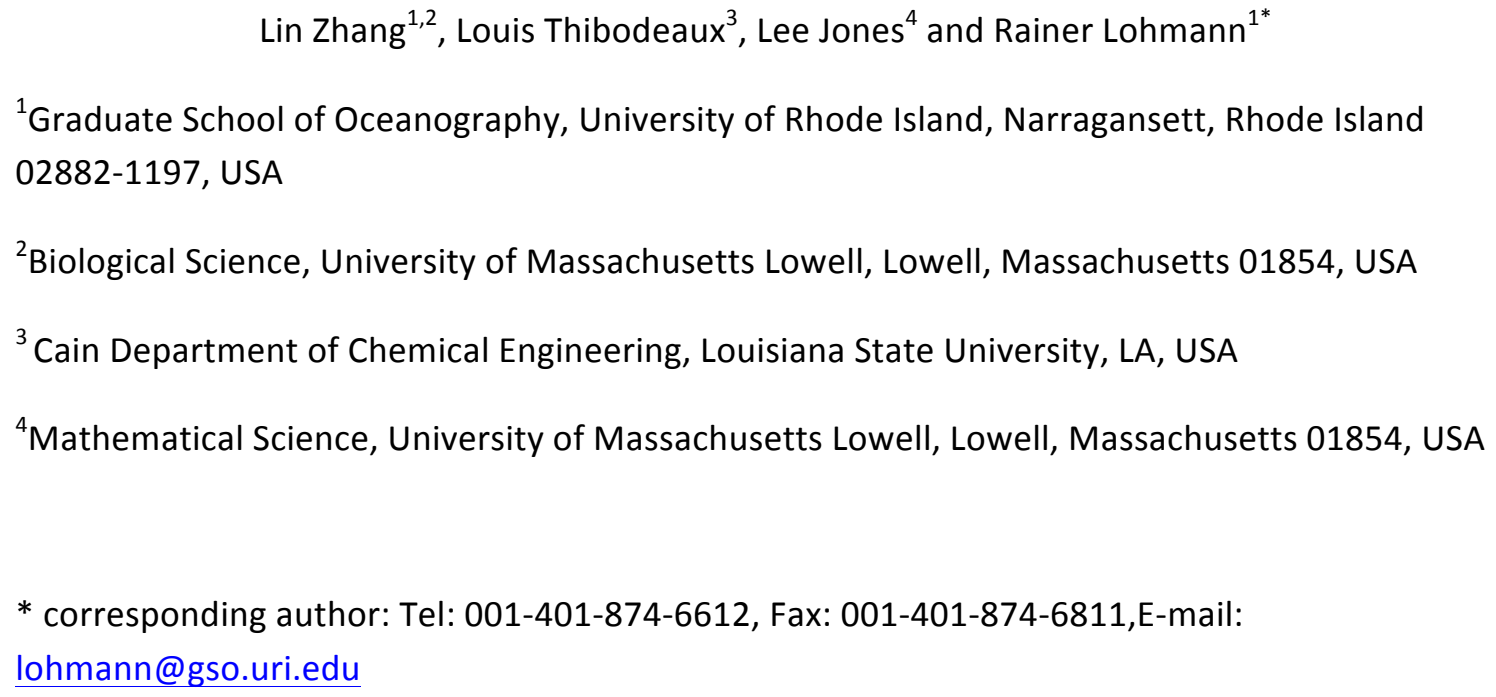

Pages: 16

Tables: 8

Figures: 2 
SI Tables

Table SI- 1. Amount of Blanks for PCBs in Air, Water, and Zooplankton Samples. Recoveries for ${ }^{13}$ C-PCB Surrogates.

\begin{tabular}{|c|c|c|c|c|c|c|c|c|c|c|}
\hline $\begin{array}{c}\text { Average } \\
\text { Blanks (pg) }\end{array}$ & PCB8 & PCB18 & PCB28 & PCB52 & PCB44 & PCB66 & $\begin{array}{c}\text { PCB } \\
101\end{array}$ & $\begin{array}{c}\text { PCB } \\
118\end{array}$ & $\begin{array}{c}\text { PCB } \\
153\end{array}$ & $\begin{array}{r}\text { PCE } \\
138\end{array}$ \\
\hline Air Blanks & 76.3 & 54.1 & 119.8 & 138.4 & 84.4 & 154.0 & 185.0 & 95.3 & 128.7 & 97.4 \\
\hline S.D. & 14.0 & 23.9 & 40.7 & 98.4 & 52.5 & 72.6 & 32.2 & 46.9 & 60.4 & $55 . \approx$ \\
\hline $\begin{array}{l}\text { Water } \\
\text { Blanks }\end{array}$ & 150.2 & 93.9 & 197.1 & 95.1 & 51.6 & 113.3 & 106.8 & 35.4 & 76.9 & 79.2 \\
\hline S.D. & 41.0 & 33.9 & 37.0 & 26.1 & 19.1 & 42.3 & 11.2 & 10.9 & 44.8 & 26.1 \\
\hline $\begin{array}{l}\text { Zooplankton } \\
\text { Blanks }\end{array}$ & 81.8 & 59.2 & 97.3 & 62.8 & 31.2 & 21.8 & 64.9 & 34.8 & 64.8 & 43.6 \\
\hline S.D. & 13.1 & 12.4 & 17.7 & 18.4 & 22.0 & 17.7 & 34.2 & 15.8 & 17.7 & 21.4 \\
\hline $\begin{array}{c}\text { Average } \\
\text { Recoveries }\end{array}$ & $\begin{array}{c}{ }^{13} \mathrm{C}- \\
\mathrm{PCB} \\
8 \\
\end{array}$ & $\begin{array}{c}{ }^{13} \mathrm{C}- \\
\mathrm{PCB} \\
28 \\
\end{array}$ & $\begin{array}{c}{ }^{13} \mathrm{C}- \\
\mathrm{PCB} \\
52 \\
\end{array}$ & $\begin{array}{c}{ }^{13} \mathrm{C}- \\
\mathrm{PCB} \\
118 \\
\end{array}$ & $\begin{array}{c}{ }^{13} \mathrm{C}- \\
\mathrm{PCB} \\
138\end{array}$ & $\begin{array}{c}{ }^{13} \mathrm{C}- \\
\mathrm{PCB} \\
180\end{array}$ & $\begin{array}{c}{ }^{13} \mathrm{C}- \\
\mathrm{PCB} \\
209\end{array}$ & & & \\
\hline Air & $90 \%$ & $95 \%$ & $91 \%$ & $97 \%$ & $97 \%$ & $107 \%$ & $80 \%$ & & & \\
\hline S.D. & $17.0 \%$ & $19.0 \%$ & $16.1 \%$ & $16.2 \%$ & $16.3 \%$ & $21.1 \%$ & $17.1 \%$ & & & \\
\hline Water & $79 \%$ & $82 \%$ & $81 \%$ & $83 \%$ & $84 \%$ & $86 \%$ & $85 \%$ & & & \\
\hline S.D. & $8 \%$ & $10 \%$ & $10 \%$ & $10 \%$ & $11 \%$ & $12 \%$ & $9 \%$ & & & \\
\hline Zooplankton & $70 \%$ & $87 \%$ & $79 \%$ & $84 \%$ & $84 \%$ & $89 \%$ & $66 \%$ & & & \\
\hline S.D. & $20 \%$ & $20 \%$ & $18 \%$ & $20 \%$ & $21 \%$ & $25 \%$ & $23 \%$ & & & \\
\hline
\end{tabular}


Table SI- 2 . PCB Concentrations $\left(\mathrm{pg} \mathrm{m}^{-3}\right)$ in the Gas Phase on the Year-day of 2008.

\begin{tabular}{cccccccccccc}
\hline Year- & PCB & PCB & PCB & PCB & PCB & PCB & PCB & PCB & PCB & PCB & $\Sigma_{\text {ICES }}$ \\
day & 8 & 18 & 28 & 52 & 44 & 66 & 101 & 118 & 153 & 138 & PCB \\
\hline 121.5 & 72.4 & 25.7 & 28.5 & 11.9 & 7.2 & 6.1 & 9.3 & 3.4 & 5.4 & 3.4 & 61.9 \\
122.0 & 82.3 & 25.4 & 27.1 & 12.5 & 7.4 & 7.9 & 12.2 & 4.6 & 9.2 & 5.3 & 70.9 \\
122.5 & 61.6 & 17.2 & 18.1 & 10.5 & 5.6 & 6.8 & 11.3 & 4.3 & 7.7 & 4.6 & 56.4 \\
123.0 & 70.1 & 20.0 & 21.0 & 8.8 & 5.9 & 6.3 & 9.3 & 3.4 & 5.9 & 4.3 & 52.7 \\
123.5 & 18.4 & 7.1 & 9.0 & & & & & & 4.4 & & 14.3 \\
124.0 & 22.9 & 7.8 & 7.4 & 3.1 & 1.8 & 1.8 & & & 2.3 & 1.4 & 14.2 \\
125.5 & 18.4 & 7.6 & 9.9 & 7.4 & 4.1 & 4.1 & 6.4 & 2.1 & 4.0 & 2.5 & 32.4 \\
126.0 & 6.7 & 2.6 & & & & & & & & & \\
126.5 & 18.3 & 6.6 & 6.0 & & & & & & & & 6.0 \\
127.0 & 26.7 & 7.6 & 6.0 & & & & & & & & 6.0 \\
127.5 & 18.3 & 5.7 & 4.9 & & & & & & & & 4.9 \\
128.0 & 16.9 & 5.0 & 4.6 & & & & & & & & 4.6 \\
128.5 & 11.2 & 3.8 & & & & & & & & & \\
129.0 & 13.0 & 4.6 & & & & & & & & & \\
129.5 & 12.3 & 4.7 & & & & & & & & & \\
130.0 & 14.0 & 5.0 & & & & & & & & & 5.4 \\
130.5 & 22.5 & 6.5 & 5.4 & & & & & & & & \\
131.0 & & & & & & & & & & & 4.6 \\
132.0 & 11.0 & 4.1 & 4.6 & & & & & & & & 27.4 \\
135.0 & 49.5 & 17.8 & 23.0 & 4.4 & 3.2 & 2.7 & & & & & 8.8 \\
136.0 & 25.7 & 7.7 & 8.8 & & & & & & & & 6.3 \\
137.0 & 12.8 & 5.1 & 6.3 & & & & & & & & 4.4 \\
138.0 & 9.1 & 4.0 & 4.4 & & & & & & & & 2.4 \\
139.0 & 8.1 & 4.7 & 2.4 & & & & & & & & \\
140.0 & 8.9 & 3.2 & 2.6 & & & & & & & & \\
141.0 & 10.6 & 3.6 & & & & & & & & & \\
142.0 & 13.6 & 4.2 & & & & & & & & & \\
142.5 & 10.4 & 3.1 & & & & & & & & & \\
\hline
\end{tabular}


Table SI- 3. PCB Concentrations ( $\mathrm{pg} \mathrm{L}^{-1}$ ) in the Dissolved Phase on the Year-day of 2008.

\begin{tabular}{|c|c|c|c|c|c|c|c|c|c|c|c|}
\hline $\begin{array}{l}\text { Year- } \\
\text { day }\end{array}$ & PCB8 & $\begin{array}{c}\text { PCB } \\
18\end{array}$ & $\begin{array}{c}\text { PCB } \\
28\end{array}$ & $\begin{array}{c}\text { PCB } \\
52\end{array}$ & $\begin{array}{c}\text { PCB } \\
44\end{array}$ & $\begin{array}{c}\text { PCB } \\
66\end{array}$ & $\begin{array}{c}\text { PCB } \\
101\end{array}$ & $\begin{array}{c}\text { PCB } \\
118\end{array}$ & $\begin{array}{c}\text { PCB } \\
153\end{array}$ & $\begin{array}{c}\text { PCB } \\
138\end{array}$ & $\begin{array}{l}\Sigma_{\text {ICES }} \\
\text { PCB }\end{array}$ \\
\hline 123.0 & 0.85 & 0.44 & 1.24 & 1.13 & 0.51 & 1.80 & 1.32 & 0.18 & 1.30 & 0.13 & 5.29 \\
\hline 123.5 & 0.16 & 0.32 & 0.98 & 0.75 & 0.38 & 1.19 & 0.79 & 0.21 & 1.37 & & 4.11 \\
\hline 124.0 & 0.97 & 0.20 & 0.23 & 0.18 & 0.28 & 0.25 & & 0.07 & 0.24 & & 0.72 \\
\hline 125.5 & 0.73 & 0.20 & 0.63 & 0.60 & 0.21 & 1.03 & 0.71 & 0.24 & 1.12 & & 3.30 \\
\hline \multicolumn{12}{|l|}{126.0} \\
\hline 126.5 & & & & & & & & & & & \\
\hline 127.0 & & & 0.15 & 0.32 & 0.20 & 1.08 & 0.62 & 0.55 & 0.98 & & 2.62 \\
\hline 127.5 & & & 0.03 & 0.89 & 0.65 & 2.65 & 0.40 & 1.73 & 2.58 & & 5.64 \\
\hline 128.0 & 0.73 & 1.27 & 1.52 & 0.58 & 0.61 & 0.92 & 0.50 & 0.68 & 0.12 & & 3.40 \\
\hline 128.5 & 1.34 & 0.34 & & & & & & & & & \\
\hline 129.0 & 0.80 & 0.25 & & & & & & & & & \\
\hline 129.5 & 0.98 & 0.03 & & & & & & & & & \\
\hline 130.0 & 1.74 & 0.36 & & & & & & 0.37 & & & 0.37 \\
\hline 130.5 & 5.36 & 1.12 & & & & & & 0.16 & & & 0.16 \\
\hline 131.0 & 1.66 & 0.68 & & & 0.17 & & & 0.23 & 0.35 & & 0.58 \\
\hline 132.0 & 3.17 & 0.69 & 0.34 & & & & & & & & 0.34 \\
\hline 135.0 & 11.24 & 2.44 & 2.71 & 0.57 & 0.26 & 0.19 & & 0.24 & 0.04 & & 3.56 \\
\hline 136.0 & 7.10 & 1.23 & 1.04 & & & & & 0.09 & & & 1.13 \\
\hline 137.0 & 5.73 & 1.00 & 0.63 & 0.31 & & 0.01 & & 0.30 & 0.21 & & 1.45 \\
\hline 138.0 & 4.23 & 1.45 & 0.39 & & & & & & & & 0.39 \\
\hline 139.0 & 3.36 & 0.72 & & & & & & 0.05 & & & 0.05 \\
\hline 140.0 & 4.96 & 0.25 & 0.22 & & & & & 0.18 & & & 0.39 \\
\hline 141.0 & 4.68 & 0.54 & 0.23 & & & & & 0.12 & & & 0.35 \\
\hline 142.0 & 0.84 & & & & & & & & & & \\
\hline 142.5 & 13.55 & 1.66 & & & & & & 0.12 & 0.53 & & 0.65 \\
\hline
\end{tabular}


Table SI- 4. Fugacity Ratios for Various PCB Congeners as a Function of Julian Day 2008.

\begin{tabular}{|c|c|c|c|c|c|c|c|c|c|c|}
\hline $\begin{array}{c}\text { Julian } \\
\text { Day }\end{array}$ & PCB8 & $\begin{array}{c}\text { PCB } \\
18\end{array}$ & $\begin{array}{l}\text { PCB } \\
28\end{array}$ & $\begin{array}{c}\text { PCB } \\
52\end{array}$ & $\begin{array}{c}\text { PCB } \\
44\end{array}$ & $\begin{array}{c}\text { PCB } \\
66\end{array}$ & $\begin{array}{c}\text { PCB } \\
101\end{array}$ & $\begin{array}{c}\text { PCB } \\
118\end{array}$ & $\begin{array}{c}\text { PCB } \\
153\end{array}$ & $\begin{array}{r}\text { PCB } \\
138\end{array}$ \\
\hline 123.0 & 21.69 & 7.56 & 3.10 & 1.58 & 3.98 & 0.55 & 2.40 & 12.60 & 1.90 & 9.03 \\
\hline 123.5 & 29.76 & 3.66 & 1.66 & & & & & & 1.33 & \\
\hline 124.0 & 5.91 & 6.09 & 5.74 & 3.38 & 2.11 & 1.09 & & & 3.69 & \\
\hline 125.5 & 5.96 & 5.69 & 2.57 & 2.26 & 6.01 & 0.56 & 2.72 & 5.08 & 1.31 & \\
\hline \multicolumn{11}{|l|}{126.0} \\
\hline \multicolumn{11}{|l|}{126.5} \\
\hline 127.0 & & & 7.47 & & & & & & & \\
\hline 127.5 & & & 30.2 & & & & & & & \\
\hline 128.0 & 5.93 & 0.64 & 0.53 & & & & & & & \\
\hline 128.5 & 2.16 & 1.82 & & & & & & & & \\
\hline 129.0 & 4.08 & 2.84 & & & & & & & & \\
\hline 129.5 & 3.31 & 25.49 & & & & & & & & \\
\hline \multicolumn{11}{|l|}{130.0} \\
\hline 130.5 & 1.10 & 0.97 & & & & & & & & \\
\hline \multicolumn{11}{|l|}{131.0} \\
\hline 132.0 & 0.89 & 0.97 & 2.41 & & & & & & & \\
\hline 135.0 & 1.11 & 1.16 & 1.49 & 1.51 & 4.12 & 2.08 & & & & \\
\hline 136.0 & 0.92 & 1.00 & 1.49 & & & & & & & \\
\hline 137.0 & 0.56 & 0.82 & 1.76 & & & & & & & \\
\hline 138.0 & 0.54 & 0.44 & 2.00 & & & & & & & \\
\hline 139.0 & 0.60 & 1.03 & & & & & & & & \\
\hline 140.0 & 0.46 & 2.05 & 2.10 & & & & & & & \\
\hline 141.0 & 0.58 & 1.06 & & & & & & & & \\
\hline 142.0 & 4.13 & & & & & & & & & \\
\hline 142.5 & 0.21 & 0.32 & & & & & & & & \\
\hline
\end{tabular}


Table SI- 5. Air-Water Exchange Velocities (m/d) Calculated Using Three Different Wind Speed Dependency Relationships; Liss and Merlivat 1986 (LM86), Nightingale et al 2000 (N00), and Wanninkhof and McGillis 1999 (WM99).

\begin{tabular}{ccccccc}
\hline $\begin{array}{c}\text { Velocity } \\
(\mathrm{m} / \mathrm{d})\end{array}$ & & PCB 28 & & & PCB 153 \\
$\begin{array}{c}\text { Year- } \\
\text { Day }\end{array}$ & LM86 & N00 & WM99 & LM86 & N00 & WM99 \\
\hline 123.0 & 0.79 & 0.90 & 1.25 & 0.54 & 0.60 & 0.76 \\
123.5 & 0.90 & 1.04 & 1.52 & 0.61 & 0.68 & 0.89 \\
124.0 & 1.79 & 2.34 & 4.01 & 1.16 & 1.39 & 1.94 \\
125.5 & 1.58 & 1.99 & 3.37 & 1.05 & 1.24 & 1.74 \\
126.0 & 1.22 & 1.47 & 2.36 & 0.81 & 0.92 & 1.26 \\
126.5 & 0.62 & 0.71 & 0.92 & 0.44 & 0.49 & 0.59 \\
127.0 & 0.34 & 0.46 & 0.50 & 0.26 & 0.33 & 0.35 \\
127.5 & 0.14 & 0.32 & 0.33 & 0.11 & 0.24 & 0.25 \\
128.0 & 0.49 & 0.59 & 0.69 & 0.36 & 0.41 & 0.47 \\
128.5 & 0.40 & 0.51 & 0.57 & 0.30 & 0.36 & 0.40 \\
129.0 & 1.09 & 1.27 & 1.97 & 0.73 & 0.82 & 1.11 \\
129.5 & 1.89 & 2.41 & 4.11 & 1.19 & 1.40 & 1.92 \\
130.0 & 0.95 & 1.10 & 1.64 & 0.64 & 0.71 & 0.94 \\
130.5 & 1.21 & 1.45 & 2.33 & 0.80 & 0.91 & 1.24 \\
131.0 & 0.84 & 0.96 & 1.36 & 0.58 & 0.64 & 0.82 \\
132.0 & 0.99 & 1.14 & 1.71 & 0.67 & 0.74 & 0.98 \\
135.0 & 0.67 & 0.77 & 1.00 & 0.47 & 0.53 & 0.64 \\
136.0 & 0.29 & 0.42 & 0.45 & 0.22 & 0.31 & 0.33 \\
137.0 & 0.62 & 0.71 & 0.90 & 0.44 & 0.49 & 0.59 \\
138.0 & 1.19 & 1.42 & 2.26 & 0.80 & 0.90 & 1.23 \\
139.0 & 1.21 & 1.44 & 2.30 & 0.81 & 0.92 & 1.26 \\
140.0 & 1.57 & 1.98 & 3.33 & 1.02 & 1.20 & 1.66 \\
141.0 & 1.41 & 1.74 & 2.88 & 0.92 & 1.07 & 1.48 \\
142.0 & 1.48 & 1.85 & 3.09 & 0.97 & 1.13 & 1.57 \\
142.5 & 1.79 & 2.30 & 3.90 & 1.13 & 1.34 & 1.83 \\
\hline & & & & & &
\end{tabular}


Table SI- 6. PCB Concentrations $\left(\mathrm{ng} \mathrm{g}^{-1}\right)$ in the Lipids of Zooplankton on the Year-day of 2008.

\begin{tabular}{ccccccccccccc}
\hline $\begin{array}{c}\text { Year- } \\
\text { day }\end{array}$ & $\begin{array}{c}\text { lipid } \\
(\%)\end{array}$ & PCB8 & $\begin{array}{c}\text { PCB } \\
18\end{array}$ & $\begin{array}{c}\text { PCB } \\
28\end{array}$ & $\begin{array}{c}\text { PCB } \\
52\end{array}$ & $\begin{array}{c}\text { PCB } \\
44\end{array}$ & $\begin{array}{c}\text { PCB } \\
66\end{array}$ & $\begin{array}{c}\text { PCB } \\
101\end{array}$ & $\begin{array}{c}\text { PCB } \\
118\end{array}$ & $\begin{array}{c}\text { PCB } \\
153\end{array}$ & $\begin{array}{c}\Sigma_{\text {ICES }} \\
\text { PCB }\end{array}$ \\
\hline 131 & $4.67 \%$ & & 0.84 & 0.64 & 1.32 & 0.59 & 0.36 & 1.79 & & 2.52 & 6.28 \\
$135 \mathrm{a}$ & $3.09 \%$ & & 0.61 & 0.67 & 0.38 & 0.15 & 0.19 & & 0.25 & 0.67 & 1.98 \\
$135 \mathrm{~b}$ & $4.97 \%$ & 0.12 & 0.08 & 0.14 & 0.28 & 0.21 & 0.59 & 0.50 & 0.20 & 0.60 & 1.73 \\
$135 \mathrm{c}$ & $1.72 \%$ & 0.28 & 0.32 & 0.81 & & & 0.44 & & & 0.74 & 1.55 \\
136 & $4.83 \%$ & 0.11 & 0.27 & 0.24 & & & & & 0.10 & 0.10 & 0.45 \\
$137 \mathrm{a}$ & $6.15 \%$ & 0.16 & 0.61 & 0.72 & 0.40 & 0.48 & 0.46 & 0.29 & 0.22 & 0.62 & 2.26 \\
$137 \mathrm{~b}$ & $2.86 \%$ & 0.64 & 0.61 & 1.64 & 0.63 & 0.29 & 0.80 & 0.64 & 0.58 & 0.77 & 4.26 \\
$138 \mathrm{a}$ & $6.45 \%$ & 0.86 & 0.45 & 0.57 & 0.26 & 0.13 & 0.22 & 0.21 & 0.16 & 0.25 & 1.44 \\
$138 \mathrm{~b}$ & $3.17 \%$ & 0.58 & 0.71 & 1.15 & 0.84 & 0.37 & 1.01 & 0.71 & 0.70 & 1.05 & 4.45 \\
139 & $2.24 \%$ & 0.24 & 0.72 & 0.87 & 0.78 & 0.31 & 0.87 & 0.81 & 0.59 & 1.21 & 4.25 \\
$140 \mathrm{a}$ & $4.83 \%$ & 0.97 & 0.57 & 0.50 & 0.16 & 0.18 & 0.15 & & 0.11 & 0.48 & 1.25 \\
\hline
\end{tabular}




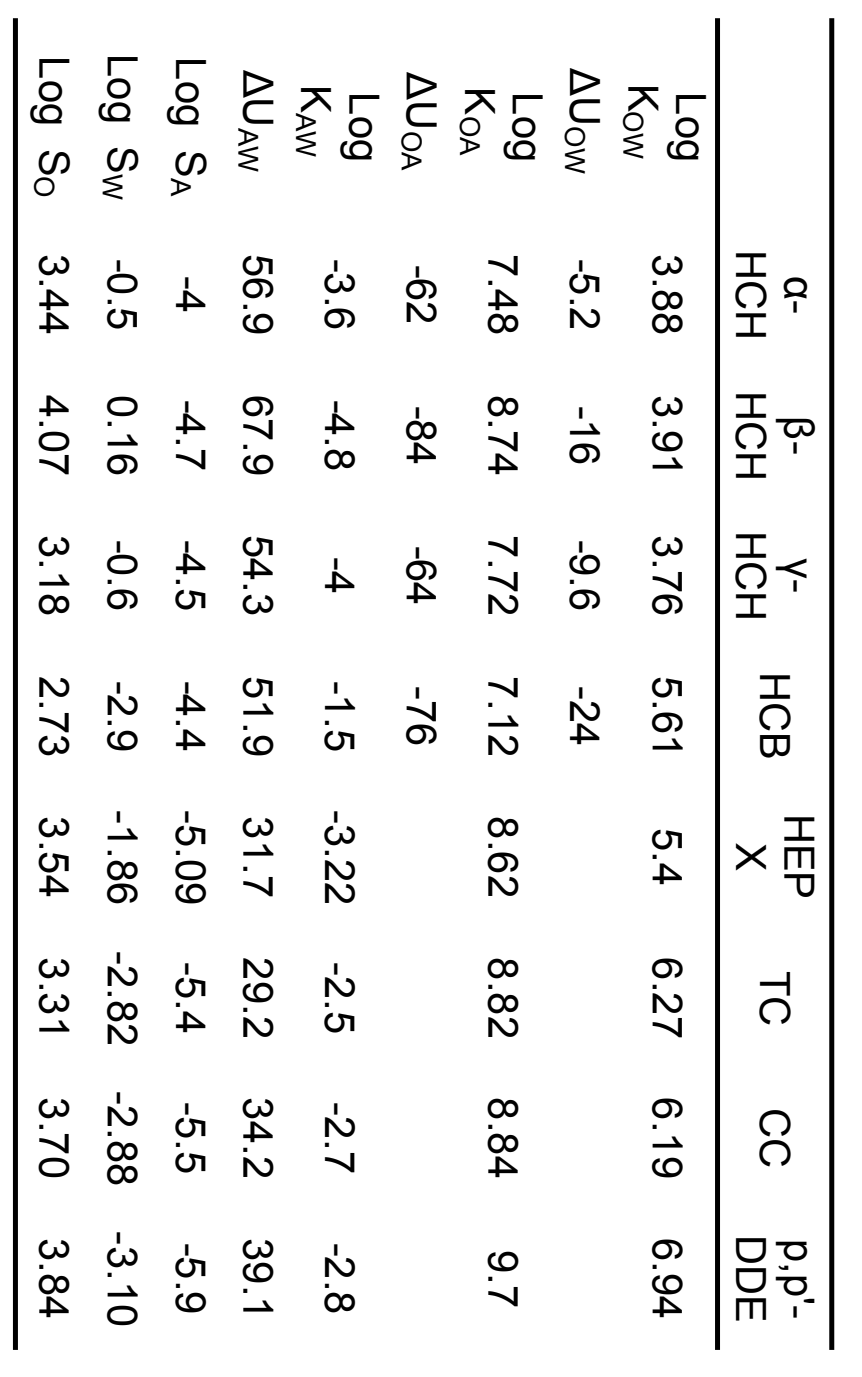

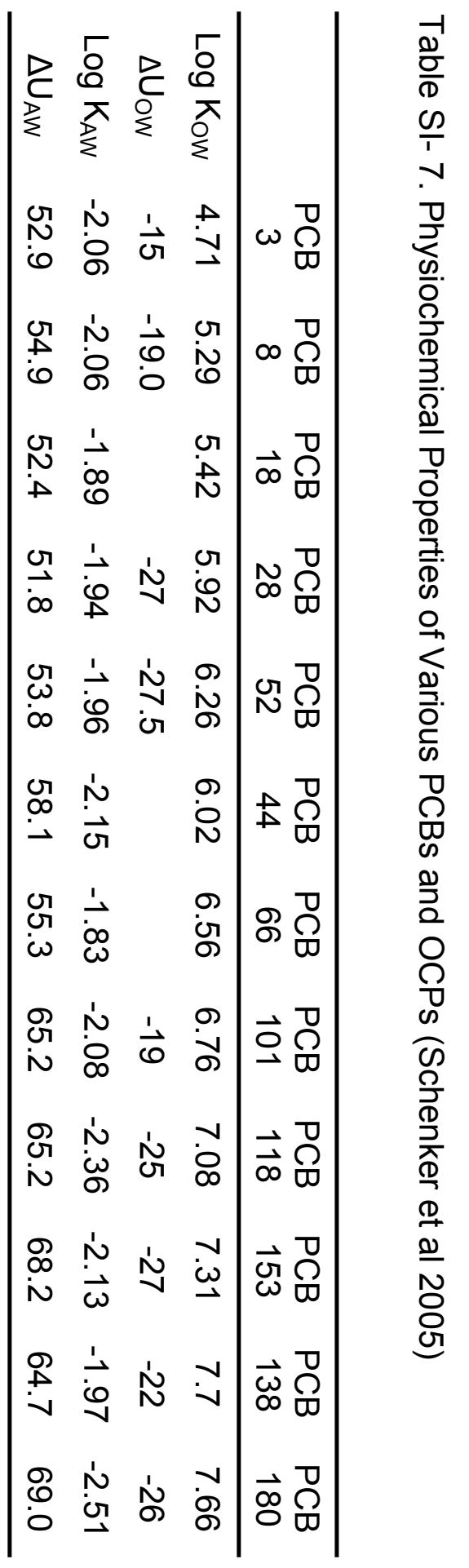


Table SI- 8. $K_{\text {lipid }}$ for different PCB congeners. Regression Slopes, Intercepts, R-Squares for log $\mathrm{K}_{\text {lipid }}$ Vs $\log \mathrm{K}_{\text {ow. }}$

\begin{tabular}{cccccccccccc}
\hline $\begin{array}{c}\text { Year- } \\
\text { day }\end{array}$ & $\begin{array}{c}\text { PCB } \\
8\end{array}$ & $\begin{array}{c}\text { PCB } \\
18\end{array}$ & $\begin{array}{c}\text { PCB } \\
28\end{array}$ & $\begin{array}{c}\text { PCB } \\
52\end{array}$ & $\begin{array}{c}\text { PCB } \\
44\end{array}$ & $\begin{array}{c}\text { PCB } \\
66\end{array}$ & $\begin{array}{c}\text { PCB } \\
118\end{array}$ & $\begin{array}{c}\text { PCB } \\
153\end{array}$ & $\begin{array}{c}\text { Slope } \\
\text { cept }\end{array}$ & $\begin{array}{c}\text { inter } \\
\text { square }\end{array}$ \\
\hline 131 & & 6.09 & & & 6.55 & & & 6.86 & 0.38 & 4.04 & 0.92 \\
$135 \mathrm{a}$ & & 5.39 & 5.39 & 5.83 & 5.77 & 5.99 & 6.03 & 7.21 & 0.79 & 0.74 & 0.74 \\
$135 \mathrm{~b}$ & 4.04 & 4.51 & 4.72 & 5.69 & 5.92 & 6.49 & 5.93 & 7.16 & 1.29 & -2.82 & 0.82 \\
$135 \mathrm{c}$ & 4.40 & 5.11 & 5.47 & & & 6.36 & & 7.25 & 1.26 & -2.29 & 0.97 \\
136 & 4.21 & 5.34 & 5.37 & & & & 6.05 & & 0.77 & 0.51 & 0.70 \\
$137 \mathrm{a}$ & 4.45 & 5.78 & 6.06 & 6.11 & & 7.49 & 5.87 & 6.46 & 0.69 & 1.54 & 0.37 \\
$137 \mathrm{~b}$ & 5.05 & 5.79 & 6.42 & 6.30 & & 7.73 & 6.29 & 6.56 & 0.64 & 2.16 & 0.39 \\
$138 \mathrm{a}$ & 5.31 & 5.50 & 6.16 & & & & & & 1.22 & -1.35 & 1.00 \\
$138 \mathrm{~b}$ & 5.13 & 5.69 & 6.47 & & & & & & 1.78 & -4.52 & 0.96 \\
139 & 4.86 & 6.00 & & & & & 7.06 & & 0.97 & 0.04 & 0.80 \\
$140 \mathrm{a}$ & 4.69 & 6.46 & 6.60 & & & & 6.53 & & 0.62 & 2.28 & 0.31 \\
\hline
\end{tabular}


Table SI- 9 Summary of Sampling Information for Air and Water Samples Collected

\begin{tabular}{|c|c|c|c|c|c|c|c|c|c|}
\hline $\begin{array}{c}\text { Julian } \\
\text { day }\end{array}$ & Latitude & Longitude & $\begin{array}{c}\text { Air } \\
\text { volume } \\
\left(\mathrm{m}^{3}\right)\end{array}$ & $\begin{array}{c}\text { Water } \\
\text { volume } \\
(\mathrm{L})\end{array}$ & Sample Date & $\begin{array}{l}\text { Start } \\
\text { Time } \\
\text { (GMT) }\end{array}$ & $\begin{array}{l}\text { End } \\
\text { Time } \\
(\mathrm{GMT})\end{array}$ & $\begin{array}{c}\text { Air } \\
\text { Temp } \\
\left({ }^{\circ} \mathrm{C}\right) \\
\end{array}$ & $\begin{array}{c}\text { Water } \\
\text { Temp } \\
\left({ }^{\circ} \mathrm{C}\right)\end{array}$ \\
\hline 121.5 & 64.1 & -21.9 & 542 & & $4 / 30 / 2008$ & $19: 45$ & $8: 30$ & 4.02 & \\
\hline 122.0 & 64.1 & -21.9 & 538 & N/A & $5 / 1 / 2008$ & $8: 50$ & $20: 30$ & 6.46 & N/A \\
\hline 122.5 & 64.2 & -22.0 & 495 & & $5 / 1 / 2008$ & $20: 50$ & 9:05 & 4.41 & \\
\hline 123.0 & 62.1 & -24.6 & 520 & 760 & $5 / 2 / 2008$ & $9: 20$ & $21: 00$ & 7.06 & 9.28 \\
\hline 123.5 & 60.9 & -25.4 & 549 & 709 & $5 / 2 / 2008$ & $21: 50$ & $8: 30$ & 8.36 & 9.43 \\
\hline 124.0 & 58.7 & -25.8 & 1294 & 1189 & $5 / 3 / \sim 5 / 4 / 2008$ & $9: 25$ & $17: 05$ & 8.97 & 9.93 \\
\hline 125.5 & 60.9 & -26.7 & 922 & 759 & $5 / 4 / \sim 5 / 5 / 2008$ & $18: 10$ & $13: 10$ & 8.97 & 10.62 \\
\hline 126.0 & 60.8 & -27.1 & 364 & 726 & $5 / 5 / 2008$ & $13: 45$ & $21: 49$ & 8.99 & 9.44 \\
\hline 126.5 & 60.9 & -27.2 & 509 & 796 & $5 / 5 / 5008$ & $22: 40$ & 09:03 & 8.34 & 9.31 \\
\hline 127.0 & 60.9 & -27.4 & 512 & 960 & $5 / 6 / 2008$ & $09: 43$ & $20: 35$ & 7.93 & 9.22 \\
\hline 127.5 & 60.8 & -27.5 & 510 & 567 & $5 / 6-5 / 7 / 2008$ & $20: 59$ & $08: 38$ & 8.39 & 9.42 \\
\hline 128.0 & 61.2 & -26.5 & 541 & 931 & $5 / 7 / 2008$ & 09:03 & $20: 25$ & 9.12 & 9.61 \\
\hline 128.5 & 61.1 & -26.7 & 572 & 518 & $5 / 7-5 / 8 / 2008$ & $20: 44$ & $09: 25$ & 8.93 & 9.55 \\
\hline 129.0 & 61.1 & -25.7 & 505 & 920 & $5 / 8 / 2008$ & $09: 50$ & $21: 13$ & 8.90 & 9.92 \\
\hline 129.5 & 61.1 & -26.7 & 499 & 639 & $5 / 8-5 / 9 / 2008$ & $21: 31$ & $10: 37$ & 7.81 & 9.22 \\
\hline 130.0 & 60.9 & -27.6 & 447 & 643 & $5 / 9 / 2008$ & $11: 01$ & $21: 05$ & 8.79 & 9.26 \\
\hline 130.5 & 60.6 & -27.6 & 473 & 539 & $5 / 9-5 / 10 / 2008$ & $21: 45$ & $11: 22$ & 7.97 & 9.30 \\
\hline 131.0 & 61.3 & -26.6 & 462 & 788 & $5 / 10 / 2008$ & $11: 22$ & $21: 41$ & 8.62 & 9.62 \\
\hline 132.0 & 61.5 & -26.0 & 941 & 1015 & $\begin{array}{c}5 / 10- \\
5 / 11 / 2008\end{array}$ & $22: 10$ & $19: 35$ & 9.39 & 9.64 \\
\hline 135.0 & 61.2 & -26.3 & 899 & 871 & $\begin{array}{c}5 / 14- \\
5 / 15 / 2008\end{array}$ & $12: 52$ & $12: 29$ & 8.15 & 9.84 \\
\hline 136.0 & 61.3 & -26.3 & 815 & 1029 & $\begin{array}{c}5 / 15- \\
5 / 16 / 2008\end{array}$ & $12: 53$ & 13:01 & 8.02 & 9.78 \\
\hline 137.0 & 61.5 & -26.0 & 623 & 952 & $\begin{array}{c}5 / 16- \\
5 / 17 / 2008\end{array}$ & $16: 40$ & $16: 50$ & 8.58 & 9.81 \\
\hline 138.0 & 61.5 & -26.1 & 894 & 816 & $\begin{array}{c}5 / 17- \\
5 / 18 / 2008\end{array}$ & $17: 15$ & $15: 28$ & 8.88 & 9.85 \\
\hline 139.0 & 61.2 & -25.5 & 1145 & 755 & $\begin{array}{c}5 / 18- \\
5 / 19 / 2008\end{array}$ & $16: 49$ & $20: 00$ & 8.81 & 10.06 \\
\hline 140.0 & 61.2 & -25.7 & 1152 & 707 & $\begin{array}{c}5 / 19- \\
5 / 20 / 2008\end{array}$ & $20: 28$ & $20: 03$ & 9.16 & 9.75 \\
\hline 141.0 & 61.5 & -25.8 & 642 & 770 & $\begin{array}{c}5 / 20- \\
5 / 21 / 2008\end{array}$ & $20: 25$ & $11: 03$ & 9.02 & 9.70 \\
\hline 142.0 & 61.4 & -25.5 & 467 & 569 & $5 / 21 / 2008$ & $11: 28$ & $22: 37$ & 9.31 & 9.73 \\
\hline 142.5 & 62.8 & -24.7 & 548 & 237 & $\begin{array}{c}5 / 21- \\
5 / 22 / 2008\end{array}$ & $22: 58$ & $11: 16$ & 8.10 & 9.05 \\
\hline
\end{tabular}




\section{SI Figures}

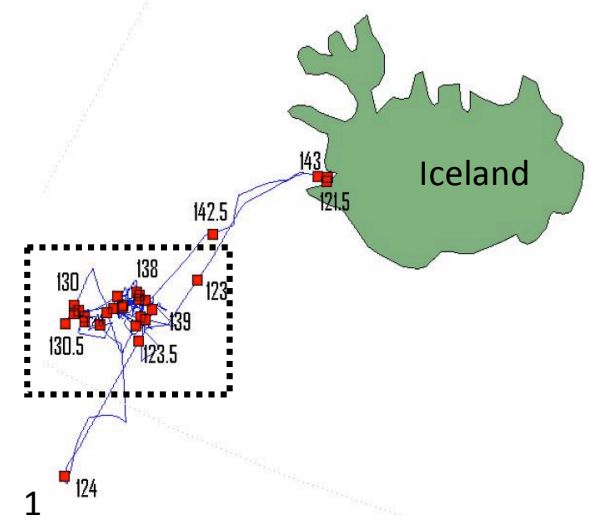

Figure SI- 1. Sampling Location. Blue lines are the ship track of R/V Knorr. Numbers are Year-day of 2008. Dashline denotes a $2^{\circ} \mathrm{X} 2^{\circ}$ box for the Eulerian model. 


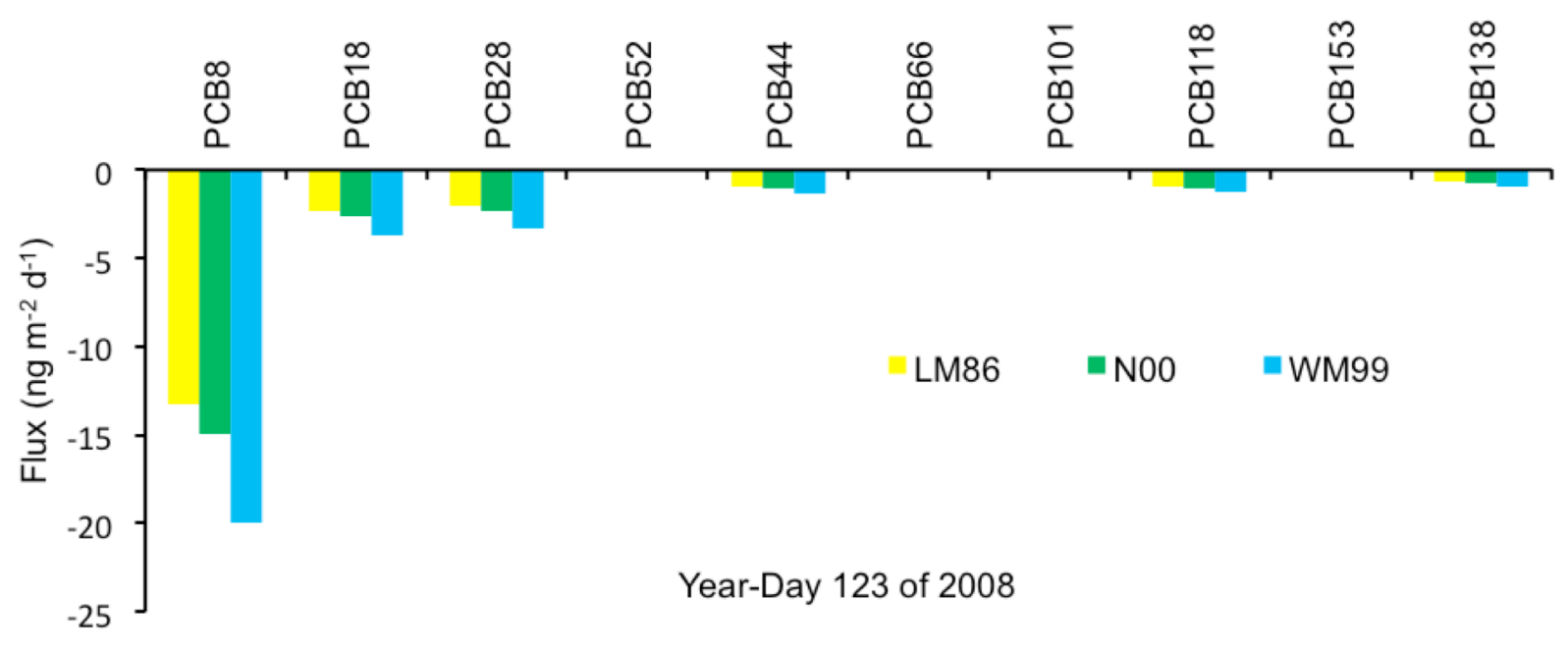

Figure SI-2. Air-Water Exchange Fluxes $\left(\mathrm{ng} \mathrm{m}^{-2} \mathrm{~d}^{-1}\right)$ of Different PCB Congeners Calculated Using Three Different Relationships on Year-Day 123 of 2008. 


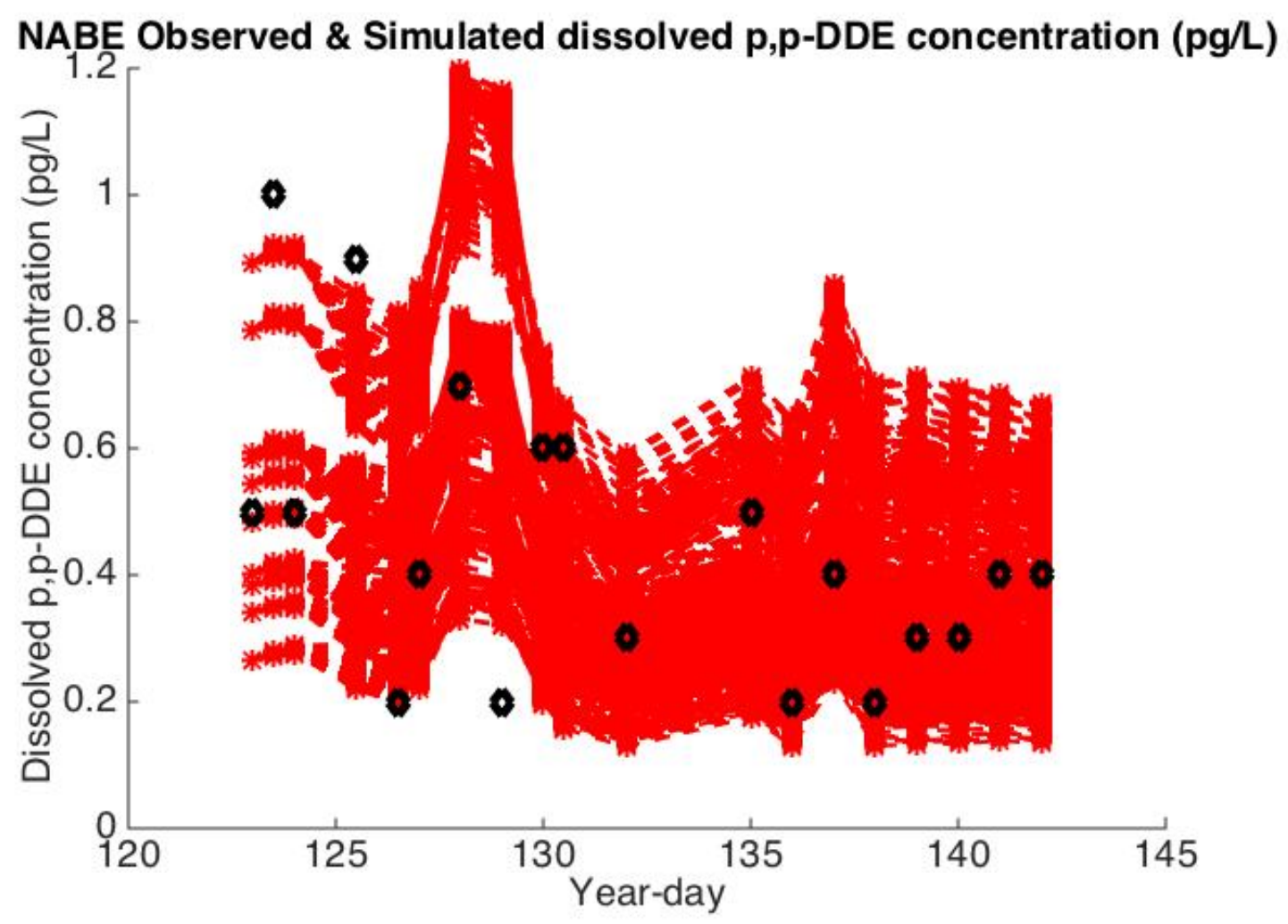

Figure SI-3, 10,000 Monte-Carlo simulation curves (red) by varying initial dissolved p,p'DDE concentrations, air-water exchange coefficients $\left(\mathrm{K}_{\mathrm{AW}}\right)$, organic carbon-water partitioning coefficients $\left(\mathrm{K}_{\mathrm{OA}}\right)$, chemical degradation constants ( $\left.\mathrm{k} \_\mathrm{deg}\right) 10$ times each following normal distribution. Observed dissolved concentrations (black) were plotted to compare with simulations. 


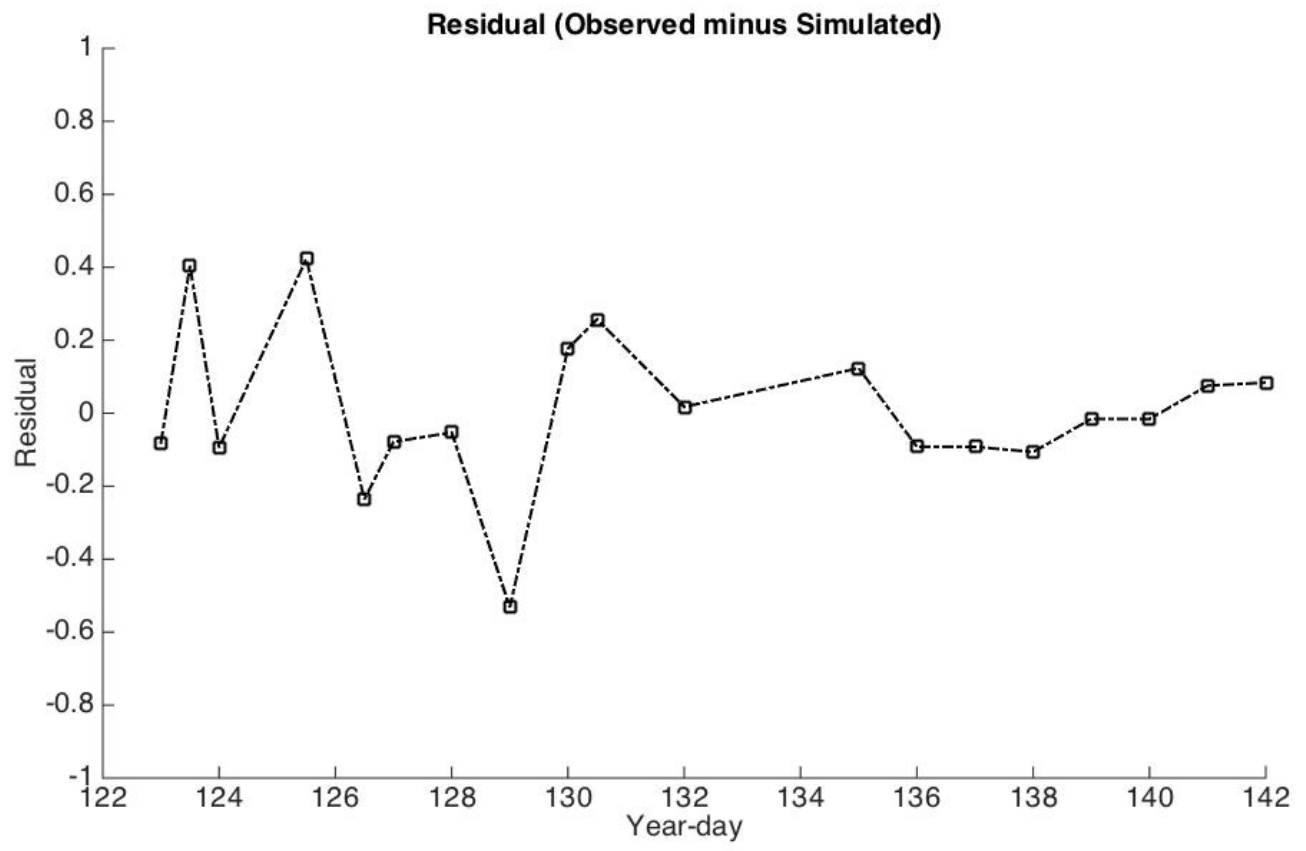

Figure SI-4 Residual (observations minus simulation) plot for dissolved p'p-DDE. 


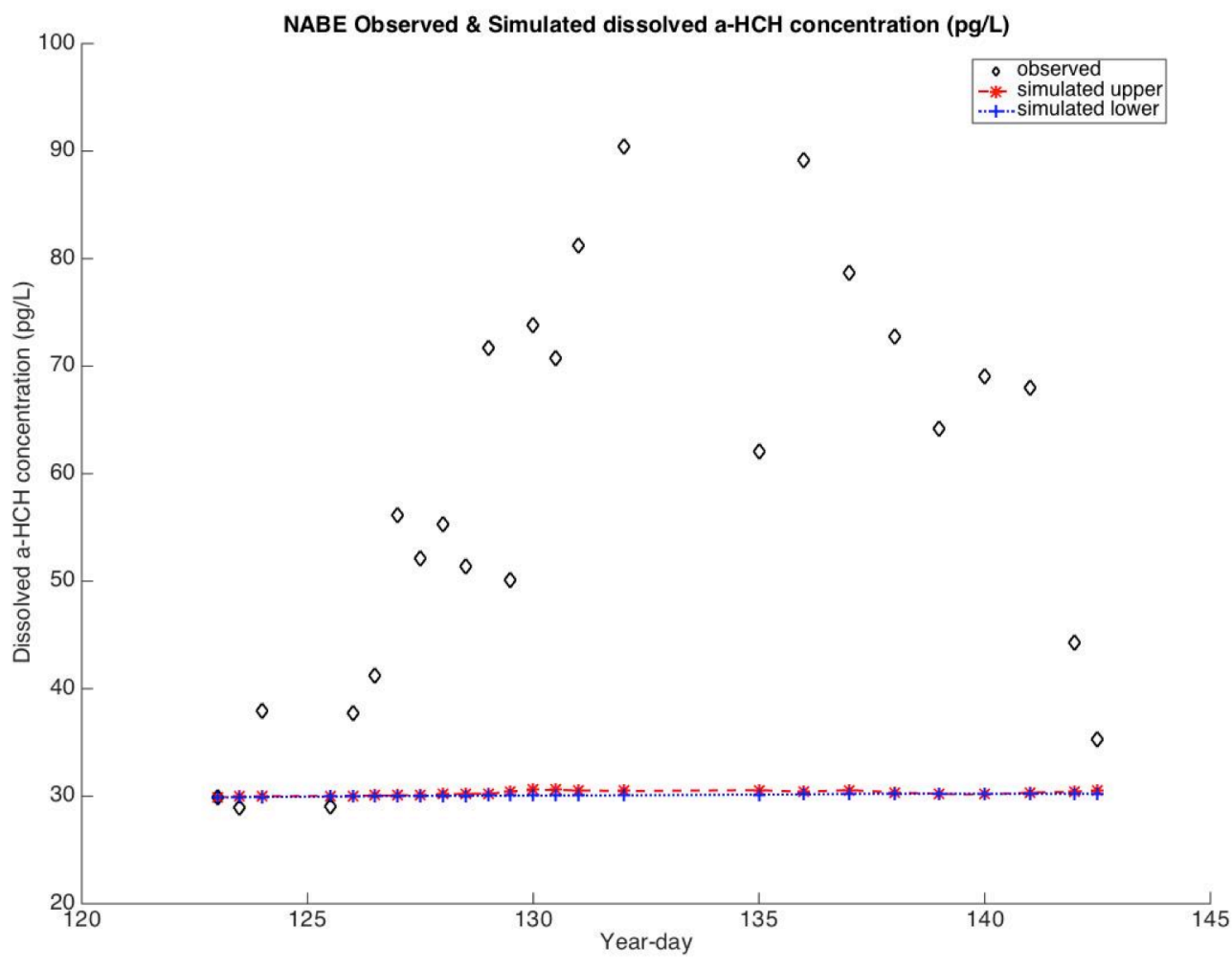

Figure SI- 5. Comparison of Modeled and Observed $\alpha-\mathrm{HCH}$ Dissolved Phase Concentrations $\left(\mathrm{pg} \mathrm{L}^{-1}\right)$ as a Function of Year-day 2008. 